\title{
Changes in the leading ENSO modes associated with the late 1970s climate shift: Role of surface zonal current
}

\author{
Soon-Il An, ${ }^{1}$ Zhengqing Ye, ${ }^{2}$ and William W. Hsieh ${ }^{2}$ \\ Received 16 April 2006; revised 11 June 2006; accepted 13 June 2006; published 22 July 2006.
}

[1] In this study, using the nonlinear principal analysis (NLPCA) technique, we demonstrated that the leading El Nino-Southern Oscillation (ENSO) mode in a physical basis changed since the late 1970s. The ENSO mode during the pre-1980s resembles the destabilized 'ocean basin mode', while during the post-1980s does 'the recharge-mode'. In particular, for the pre-1980s, the surface zonal current mainly acts as an intensifier of the ENSO, while that during post-1980s it plays a role in the transition of the ENSO cycle. The NLPCA results are reconfirmed by the eigen analysis of the linearized intermediated ENSO model. Over a reasonable parameter range, the leading eigen modes associated with the pre-1980s are completely separable from those associated with the post-1980s. The eigen structures for each decadal-period resemble the corresponding NLPCA patterns. Citation: An, S.-I., Z. Ye, and W. W. Hsieh (2006), Changes in the leading ENSO modes associated with the late 1970s climate shift: Role of surface zonal current, Geophys. Res. Lett., 33, L14609, doi:10.1029/2006GL026604.

\section{Introduction}

[2] In the late 1970s, an abrupt change of the climate state in the midlatitude as well as in the tropical Pacific was observed [Trenberth and Hurrel, 1994]. Together with such climate shift, the main characteristics of the interannual variability over the tropical Pacific have changed [Wang and An, 2001; An and Wang, 2000; An et al., 2005]. On one hand, the multi-decadal variation in the climate state causes the non-stationary characteristics of El Niño-Southern Oscillation (ENSO) [e.g., An and Jin, 2000]. On the other hand, it can also be attributed to the internal nonlinear process of ENSO in a certain parameter regime [Timmermann et al., 2003; An and Jin, 2004]. Although it is still uncertain whether the ENSO system is linear or nonlinear [e.g., Philander and Fedorov, 2003; An and Jin, 2004], we view that the late 1970s climate shift is fairly enough to change the leading ENSO mode.

[3] Recently, An et al. [2005] obtained the nonlinear ENSO cycle in the evolution of tropical Pacific thermocline anomalies using the nonlinear principal component analysis (NLPCA), which fits a closed curve to the data in the multidimensional PC (principal component) space. Interestingly, the shape of the closed curve, especially its asymmetry, significantly changed since the late 1970s, inferring a

\footnotetext{
${ }^{1}$ Department of Atmospheric Sciences/Global Environment Laboratory, Yonsei University, Seoul, Korea.

${ }^{2}$ Department of Earth and Ocean Sciences, University of British Columbia, Vancouver, British Columbia, Canada.
}

Copyright 2006 by the American Geophysical Union. 0094-8276/06/2006GL026604\$05.00 change of the leading ENSO mode. Furthermore, An and Jin [2000] showed that the interdecadal change could lead to a quantitative change in the leading eigen mode of a stripped-down version of the intermediate ENSO model. As a further study over the previous works, here we present observational evidence on the interdecadal change in the leading ENSO mode and perform an eigen analysis of the intermediate complexity ENSO model.

\section{Combined NLPCA of the Sea Level and Current Anomalies}

[4] We have applied the combined principal component analysis (CPCA; a.k.a. Multi Singular Value Decomposition method) to monthly mean sea level height, sea surface temperature (SST) and upper-50m surface zonal current anomalies. The anomalies were obtained by removing the climatological monthly mean, and then a 7-point running mean was applied. The data utilized are the University of Maryland Simple Ocean Assimilation (SODA) data for January 1958-December 2001 [Carton et al., 2000]. In Figure 1, the main features of sea level height, SST and surface current's variability are summarized by the two leading modes: the first and second modes of sea level height are a zonal contrast mode (Figure 1c) and an approximate zonal-symmetric mode (Figure 1d) with respect to the equator, respectively; those of SST are a horseshoe pattern like the mature phase of ENSO (Figure 1a) and an equatorially-confined pattern like the initial phase of ENSO (Figure 1b), respectively; those of the surface current are a localized zonal/meridional contrast mode (Figure 1e) and an equatorial basin-wide mode (Figure 1f), respectively. Obviously, the first leading mode is highly correlated to the ENSO index, and the second mode shows a lead/lag relationship to the ENSO index.

[5] The corresponding trajectory plot of the two leading PCs associated with the sea level height is shown in Figure 1g. The virtual center of the tracks for the pre-1980s (19581979 ) is located at the origin, while that for the post-1980s (1980-2001) moves away from the origin, inferring a possible regime shift in the behavior of the two PCs.

[6] From the first six leading CPCA modes, we extracted the combined NLPCA modes; that is, a closed curve (based on a neural network representation; see Hsieh [2004] for details) is used to fit the data in the 6-dimensional PC space (similar to An et al. [2005]). In Figure 2, the evolution features of SST, sea level height and surface zonal current associated with the first NLPCA mode on the equatorial band are shown. These modes explain $65.7 \%$ and $73.3 \%$ of total variance (of the 6 leading modes only, not of the original data set) during the pre- and post-1980s, respectively, which are greater than those of the leading CPCA 
(a) SST (mode $1,36 \%)$

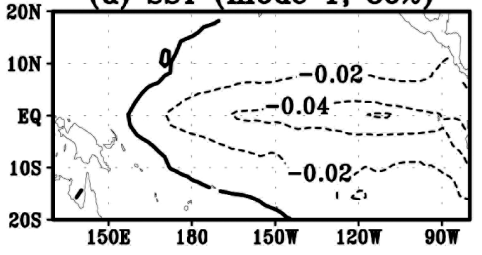

(b) SST (mode $2,14 \%$ )
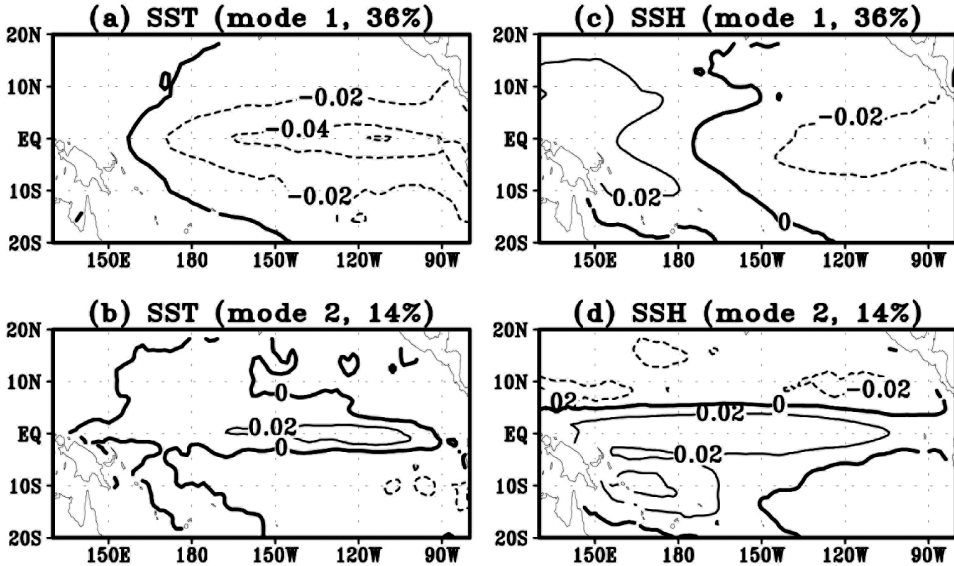

(d) SSH (mode $2,14 \%$ )
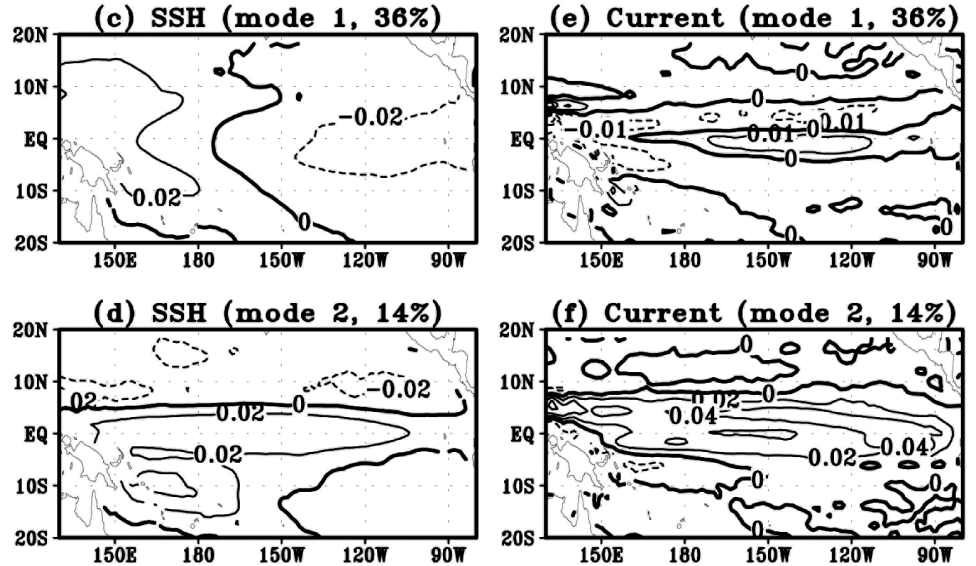

(g) Trajectory

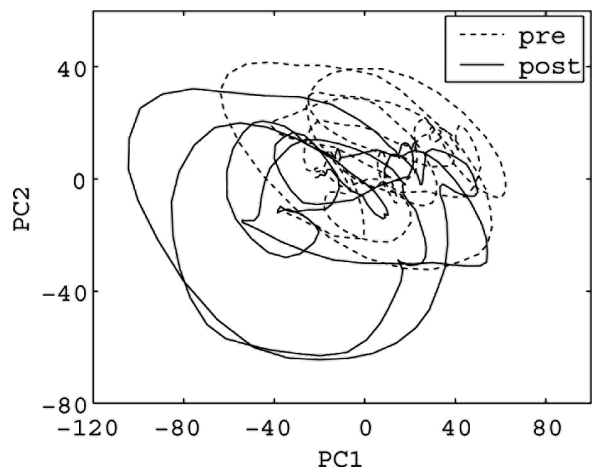

Figure 1. $(\mathrm{a}-\mathrm{f})$ The first and second principal component spatial pattern of (left) the SST, (middle) sea level height and (right) surface zonal current anomalies obtained from the Combined Principal Component Analysis (CPCA). The percentage of variance explanation appears at the top of each plot. (g) Trajectory plot of the principal components of the first and second PCs.

mode (the CPCA mode 1 accounts for $49.5 \%$ and $56.3 \%$ of the total variance from the 6 leading PC2 during the preand post-1980s, respectively). For the pre-shift regime, the standing-type oscillation in the sea level height with its node around the dateline and its center at the $120^{\circ} \mathrm{W}$ is observed; the surface zonal current also shows a standing-type oscillation with a basin-wide pattern and its minimum at boundaries; and SST anomalies shows a westward propagating (a) SST (mode 1, 65.7\%)

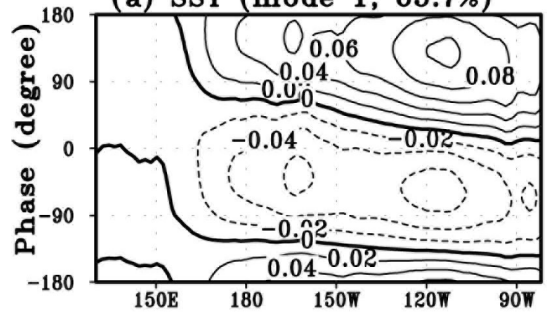

(d) SST (mode 1, 73.3\%)

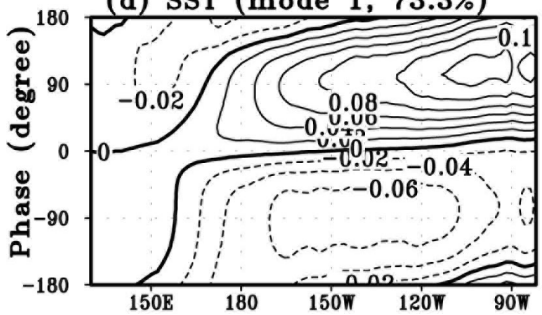

(b) SSH (mode 1, 65.7\%)

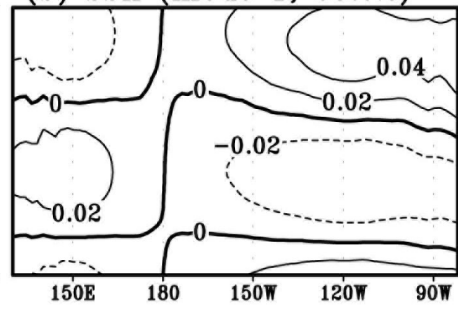

(e) SSH (mode 1,73.3\%)

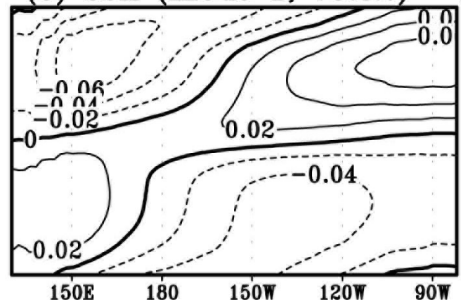

(c) Current (mode 1, 65.7\%)

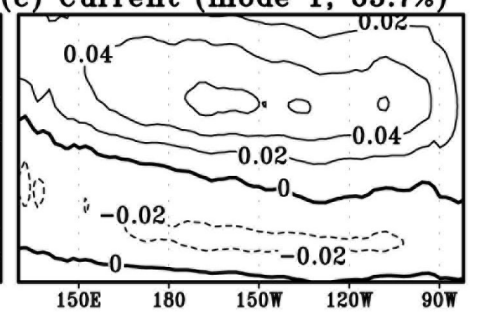

(f) Current (mode 1, 73.3\%)

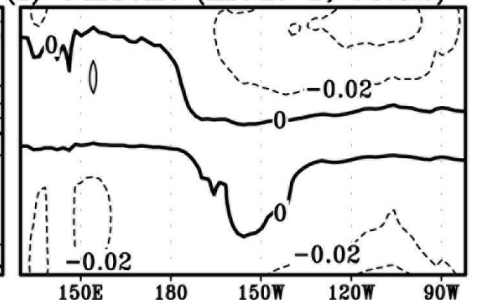

Figure 2. Phase-longitude section of (left) the SST, (middle) sea level height, and (right) the surface zonal current anomalies over the equatorial band $\left(5^{\circ} \mathrm{S}-5^{\circ} \mathrm{N}\right)$ associated with the first NLPCA mode $(\mathrm{a}-\mathrm{c})$ for the pre-1980s and $(\mathrm{d}-\mathrm{f})$ for the post-1980s. Phase generally increases with time. 
tendency, especially in the eastern-to-central Pacific. The surface zonal current pattern is very similar to the so-called 'ocean basin mode' [Neelin and Jin, 1993]. The ocean basin mode is a natural mode produced by the equatorial trapped waves and their reflection at boundaries: the equatorial Kelvin wave travels eastward until it hits the eastern boundary where it is mostly reflected to the equatoriallytrapped Rossby wave; this Rossby wave moves westward and reflects at the western boundary as the Kelvin wave; thus a semi-closed loop completed by the traveling-waves with 8-12 month periods is established in the tropical Pacific basin (with energy loss at the eastern boundary due to the poleward traveling coastal Kelvin wave [Jin, 1997]). Neelin and Jin [1993] mentioned that this ocean basin mode can be destabilized by the zonal advective feedback (i.e., zonal advection of the mean zonal temperature gradient by the anomalous zonal current). Once the ocean basin mode becomes unstable, it could be modified as the interannual mode, and have larger amplitude than the uncoupled ocean basin mode. An and Jin [2000] showed that the zonal temperature gradient of the mean SST during the 1961-75 is relatively larger than that during 1981-95, and it caused the destabilization of the ocean basin mode during the pre-1980s. In many respects, the leading NLPCA mode of the observations is consistent with the previous study.

[7] For the post-1980s, the evolution features are quite different from those for the pre-1980s (bottom plots in Figure 2). The standing feature in the sea level height turns to the propagating feature; the basin-wide pattern of the surface current disappears; and the westward moving tendency of SST changes to the eastward moving tendency [e.g., An and Wang, 2000]. The maximum surface current in the eastern Pacific appears when the zonal gradient of the sea level height is at maximum, indicating that the surface current is mostly due to the local pressure gradient from the mass difference rather than the surface wind stress. For example, the positive SST anomaly and the resulting convective atmospheric heat generate the positive wind stress anomaly in the western part of the center of the SST anomaly, which may enforce the westerly zonal surface current. But the zonal current anomaly is negative as shown here. Since the geostrophic current can be represented by the gradient of the sea level height (or thermocline), the effect of the zonal current in inducing the coupled mode is easily adding into the effect of sea level height. An and Jin [2001], under the two-box approximation of the intermediate ENSO model, theoretically proved this point, and mentioned that the two-box approximation could represent the ocean adjustment but not the ocean wave dynamics, thus only the recharge mode is possible. In this regard, the ENSO mode inferred from the NLPCA pattern for the post-shift regime is presumably the recharge mode due to the destabilization of the ocean adjustment mode [Jin, 1997]. Note that the life time and amplitude for the positive zonal current anomaly and those for the negative zonal current anomaly are not the same. This asymmetric oscillation was dominant during the post-1980s [An et al., 2005].

[8] The phase relationships among the SST, sea level height, and surface zonal current appeared in the NLPCA mode give an idea on the role of the surface current for each decadal period. For the pre-1980s, as shown in Figure 2, the

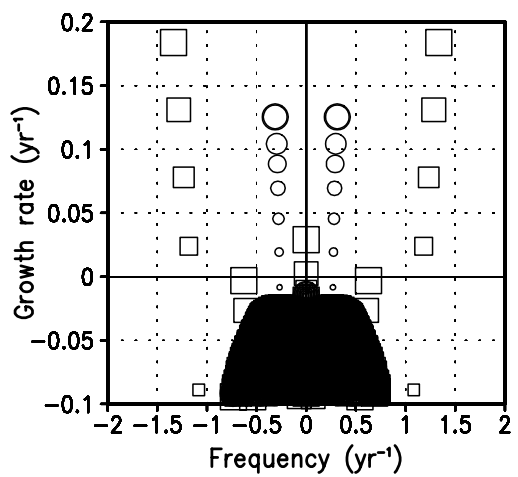

Figure 3. Eigenvalues obtained from the linearized intermediate ENSO model for the pre-1980s background condition (open square), and for the post-1980s background condition (open circle). The eigen spectrum is symmetric around the zero frequency. The size of marker indicates the strength of the coupling coefficient $(\mu)$.

sea level height anomalies over the equatorial eastern Pacific are almost in-phase with the surface zonal current anomalies. Since a positive (negative) surface zonal current anomaly generates a positive (negative) SST anomaly through the warm (cold) surface water advection by the eastward (westward) zonal current, the surface current plays a role in the intensification of this system. For the post1980s, on the other hands, the surface zonal current anomalies in the equatorial Eastern Pacific lead the sea level height anomalies by about a quarter of a cycle. It infers that the surface zonal current plays a role of the transition mechanism in this oscillation. Thus, for each decadal period, the role of the surface zonal current in ENSO evolution is quite distinctive.

\section{Eigen Analysis of the Intermediate ENSO Model}

[9] In the previous section, we presented the observational evidence for the decadal changes of the leading ENSO mode. Here, we perform the eigen analysis of the intermediate ENSO model linearized with respect to the climatological mean background states (for details, see $A n$ et al. [2004]). The background states for the model are specified based on the observed. To avoid model drifts away from the original background state of the intermediate model when the climate states for 1981-95 or 1961-75 are directly used, we add/subtract the difference between the annual-mean state for 1981-95 and that for 1961-75 into/ from the original background state, which includes surface wind, atmospheric surface divergence, SST, current, upwelling, and thermocline depth (approximately $20^{\circ} \mathrm{C}$ isotherm depth). For this calculation, again SODA data are utilized.

[10] Here, the eigenvalues obtained from the linearized intermediate ENSO model for each background state are plotted in Figure 3. To test the model behaviour for different parameters, the eigen analysis is performed for various values of the coupling coefficient, $\mu$. The coupling coefficient, representing the strength of the dynamical/thermodynamical coupling between atmosphere and ocean in the 
(a) SST_6175

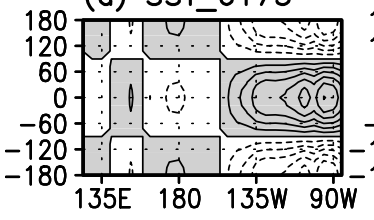

(d) SST_8195

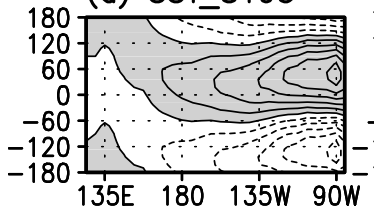

(b) H_6175

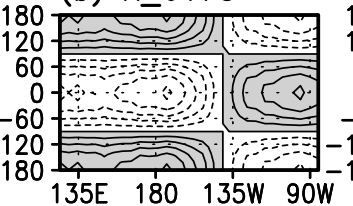

(e) H_8195

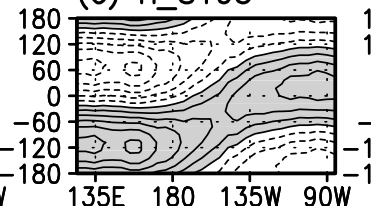

(c) Us_6175

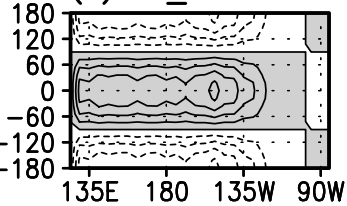

(f) Us_8195

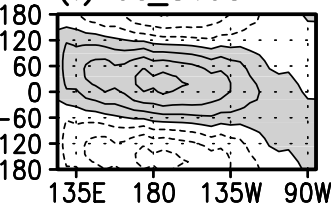

Figure 4. Time-longitude dependence of the leading eigen mode (growth rate suppressed) along the equator $\left(5^{\circ} \mathrm{S}-5^{\circ} \mathrm{N}\right)$ for $\mu=1.0$ : (a) SST, (b) height, and (c) surface zonal current for the pre-1980s condition; (d-f) as in Figure 4a-4c except for the post-1980s condition. Y-axis indicates degrees in one cycle. The contour intervals of SST, height, and current for the pre-1980s (the post-1980s) condition are $0.1 \mathrm{C}(0.5 \mathrm{C}), 1 \mathrm{~m}(5 \mathrm{~m})$, and $1 \mathrm{~cm} \mathrm{~s}^{-1}\left(5 \mathrm{~cm} \mathrm{~s}^{-1}\right)$, respectively.

physical sense, is also a control parameter for the Hopfbifurcation. The Hopf-bifurcation occurs at the boundary between stable and unstable regimes. A striking feature in Figure 3 is the clear separation between the leading eigen modes for the post-1980s and those for the pre-1980s. This distinction is maintained even if the coupling coefficient changes. For the post-1980s, the leading eigen mode for $\mu=$ 1 has a frequency of about $0.3 \mathrm{yr}^{-1}$ ( $\sim 3$ years period). As the coupling coefficient increases, the growth rate significantly increases, and the frequency increases by a tiny amount. Over the range of the coupling coefficient $(0.8<$ $\mu<1.4$, with $\mu=1$ as the standard value), the leading modes are unstable oscillatory modes with interannual period. The Hopf-bifurcation point is located between $\mu=0.8$ and 0.9 . The leading mode corresponds to the recharge mode [ $\mathrm{Jin}$, 1997].

[11] For the pre-1980s, the Hopf-bifurcation, on the other hands, is observed between $\mu=1.3$ and 1.4. The leading modes in the sub-critical regime are a damped stationary mode, while those in the supercritical regime are the highfrequency westward-propagating oscillatory mode, resembling the 'SST mode' [Jin and Neelin, 1993]. When $\mu>1.6$, the second leading modes with the quasi-biennual period are brought up. This quasi-biennual mode becomes unstable for $\mu>1.7$.

[12] To make the point more clearly, Figure 4 shows the eigen structure of SST, height (the height of this dynamic model can be a proxy of the thermocline depth), and surface zonal currents associated with the leading eigen modes for the standard value of the coupling coefficient $(\mu=1)$. As mentioned previously, the leading mode for the pre-1980s is a damped stationary mode. The SST anomalies over the eastern Pacific are positively correlated with both the thermocline depth and surface zonal current anomalies in the spatial pattern, consistent with the NLPCA mode of the observations (Figure 2). As mentioned before, physically, both the positive (negative) thermocline depth anomaly and the positive (negative) surface zonal current anomaly induce the positive (negative) SST anomaly, through vertical and zonal thermal advection, respectively. Thus, as in the NLPCA result, the surface zonal current plays a role in the intensification of the SST anomaly. However, the rigid in-phase relationship of this stationary mode is not realistic.
More realistic modes can be captured in other parameter ranges, as discussed in the next section.

[13] For the post-1980s, the eigen structure of the leading mode (about 3-year period) resembles the observed mode from NLPCA (3.7-year period). The SST maximum is confined over the eastern Pacific, and the evolution of the thermocline depth anomaly is similar to the observed. In the eastern Pacific, the surface zonal current anomaly leads the SST anomaly by a quarter cycle, indicating that the zonal current anomaly plays a transition role in this oscillatory mode. The phase relationship among three variables infers that the SST anomaly over the eastern Pacific is closely related to the thermocline depth anomaly and that over the central Pacific is related to the surface zonal current anomaly. This phase relationship has been observed in the NLPCA mode (see Figures 2d-2f). Overall, the eigen structure of the leading mode obtained from the linearized intermediate ENSO model is consistent with the NLPCA result, when the corresponding background climate state is accounted for. Thus, the observed evidence for the change in the leading mode associated with the late 1970s climate shift is reconfirmed by the eigen analysis of the dynamical system.

\section{Concluding Remarks}

[14] In this study, we proposed that the decadal change in the ENSO characteristics was rather the change of the leading eigen mode driven by the different physical consequence than that addressed by a statistical artifact. As a physical consequence, the role of the surface zonal current is clearly distinct in the NLPCA modes associated with the two regimes: for the pre-1980s, the surface zonal current tends to be an intensifier of the system, while that for the post-1980s plays a role in the ENSO transition. The eigen analysis of the intermediate ENSO model reconfirmed this point such that over a reasonable parameter range (here, the coupling coefficient is varied), the possible leading eigen mode associated with the pre-1980s is completely separable from that associated with the post-1980s. All these results provide firm support for the decadal change of the leading ENSO mode.

[15] One may argue that the leading eigen mode associated with the pre-1980s for $\mu=1$ is unrealistic so that it is 
not matched to the observations. A possible explanation for this discrepancy is: Since we do not know the right value of the standard parameter (e.g., the coupling coefficient), our choice may not always be correct. For example, as shown in Figure 3, the leading eigen mode by changing the coupling strength $(\mu)$ relocates the stationary regime to the oscillatory regime. In the oscillatory regime, although the period of the leading eigen mode is slightly shorter than the observed, the eigen structures of SST, thermocline depth, and zonal current resembles the observed (not shown). In addition, the mean vertical stratification of the ocean and the contributions by the high-order baroclinic modes, which could lead to change in the characteristics of the ENSO mode [Moon et al., 2004], are not considered here.

[16] We have noticed a sort of inconsistency between the observed surface zonal current and that of the eigenstructure over the central Pacific (Figures $2 \mathrm{f}$ and $4 \mathrm{f}$ ), particularly in their evolution features with respect to the evolution of SST anomaly. We concluded that it is due to the model's deficiency for simulating the undercurrent. As we know, strong zonal flow, the so-called 'undercurrent', exists in the equatorial subsurface. The undercurrent may influence the surface zonal current through the momentum diffusion or nonlinear momentum flux [Philander, 1990]. The undercurrent is weaker during the El Nino year. So, the anomalous undercurrent becomes negative. This anomalous easterly momentum is reflected to the surface zonal current, thus the surface zonal current anomaly tends to be negative as well. The model could not capture this phenomenon.

[17] Acknowledgments. S.-I. An is supported by the SRC program of Korea Science and Engineering Foundation, and the Brain Korea 21 project. Z. Ye and W. Hsieh are supported by the Natural Sciences and Engineering Research Council of Canada.

\section{References}

An, S.-I., and F.-F. Jin (2000), An eigen analysis of the interdecadal changes in the structure and frequency of ENSO mode, Geophy. Res. Lett., 27, 1573-1576.
An, S.-I., and F.-F. Jin (2001), Collective role of thermocline and zonal advective feedbacks in the ENSO mode, J. Clim., 14, 3421-3432.

An, S.-I., and F.-F. Jin (2004), Nonlinearity and asymmetry of ENSO, J. Clim., 17, 2399-2412.

An, S.-I., and B. Wang (2000), Interdecadal change of the structure of the ENSO mode and its impact on the ENSO frequency, J. Clim., 13, 20442055.

An, S.-I., A. Timmermann, L. Bejarano, F.-F. Jin, F. Justino, Z. Liu, and A. W. Tudhope (2004), Modeling evidence for enhanced El NiñoSouthern Oscillation amplitude during the Last Glacial Maximum, Paleoceanography, 19, PA4009, doi:10.1029/2004PA001020.

An, S.-I., W. W. Hsieh, and F.-F. Jin (2005), A nonlinear analysis of the ENSO cycle and its interdecadal changes, J. Clim., 18, 3229-3239.

Carton, J., G. Chepurin, X. Cao, and B. Giese (2000), A simple ocean data assimilation analysis of the global upper ocean 1950-95. part I: Methodology, J. Phys. Oceanogr., 30, 294-309.

Hsieh, W. W. (2004), Nonlinear multivariate and time series analysis by neural network methods, Rev. Geophys., 42, RG1003, doi:10.1029/ 2002RG000112.

Jin, F.-F. (1997), An equatorial ocean recharge paradigm for ENSO. part II: A stripped-down coupled model, J. Atmos. Sci., 54, 811-829.

Jin, F.-F., and J. D. Neelin (1993), Modes of interannual tropical oceanatmosphere interaction-A unified view. part I: Numerical results, J. Atmos. Sci., 50, 3477-3503.

Moon, B., S. Yeh, B. Dewitte, J. Jhun, I. Kang, and B. P. Kirtman (2004), Vertical structure variability in the equatorial Pacific before and after the Pacific climate shift of the 1970s, Geophys. Res. Lett., 31, L03203, doi:10.1029/2003GL018829.

Neelin, J. D., and F.-F. Jin (1993), Modes of interannual tropical oceanatmosphere interaction-A unified view. part II: Analytical results in the weak-coupling limit, J. Atmos. Sci., 50, 3504-3522.

Philander, G. H. (1990), El Nino, La Nina, and the Southern Oscillation, 293 pp., Elsevier, New York.

Philander, G. H., and A. Fedorov (2003), Is El Niño sporadic or cyclic?, Annu. Rev. Earth Planet. Sci., 31, 579-594.

Timmermann, A., F.-F. Jin, and J. Abshagen (2003), A nonlinear theory for El Nino bursting, J. Atmos. Sci., 60, 152-165.

Trenberth, K. E., and J. W. Hurrel (1994), Decadal atmosphere-ocean variations in the Pacific, Clim. Dyn., 9, 303-319.

Wang, B., and S.-I. An (2001), Why the properties of El Nino changed during the late 1970s, Geophy. Res. Lett., 28, 3709-3712.

S.-I. An, Department of Atmospheric Sciences/Global Environment Laboratory, Yonsei University, 134 Shinchon-dong, Seodaemun-gu, Seoul 120-749, Korea. (sian@yonsei.ac.kr)

W. W. Hsieh and Z. Ye, Department of Earth and Ocean Sciences, University of British Columbia, 6339 Stores Road, Vancouver, BC, Canada V6T1Z4. 